\title{
Characterization of a 50kW Inductively Coupled Plasma Torch for Testing of Ablative Thermal Protection Materials
}

\author{
Benton R. Greene ${ }^{1}$, Noel T. Clemens ${ }^{2}$, and Philip L. Varghese ${ }^{3}$ \\ University of Texas at Austin, Austin, TX, 78705 \\ Stanley A. Bouslog ${ }^{4}$ and Steven V. Del Papa ${ }^{5}$ \\ NASA Johnson Space Center, Houston, TX, 77058
}

\begin{abstract}
With the development of new manned spaceflight capabilities including NASA's Orion capsule and the Space- $X$ Dragon capsule, there is a renewed importance of understanding the dynamics of ablative thermal protection systems. To this end, a new inductively coupled plasma torch facility is being developed at UT-Austin. The torch operates on argon and/or air at plasma powers up to $50 \mathrm{~kW}$. In the present configuration the flow issues from a low-speed subsonic nozzle and the hot plume is characterized using slug calorimetry and emission spectroscopy. Preliminary measurements using emission spectroscopy have indicated that the torch is capable of producing an air plasma with a temperature between $6,000 \mathrm{~K}$ and $8,000 \mathrm{~K}$ depending on the power and flow settings and an argon plasma with a temperature of approximately $12,000 \mathrm{~K}$. The operation envelope was measured, and heat flux measured for every point within the envelope using both a slug calorimeter and a Gardon gauge heat flux sensor. The torch was found to induce a stagnation point heat flux of between 90 and 225 $\mathrm{W} / \mathrm{cm}^{2}$.
\end{abstract}

\section{Introduction}

$\mathrm{D}$ eveloping effective thermal protection systems for spacecraft entering a planetary atmosphere is a challenging task. The system must be as light as possible while still effectively dissipating the heat load of atmospheric entry and insulating the spacecraft against it. Additionally, flight testing such a system is prohibitively expensive if not impossible, and the designers must be almost certain the system will work before it is flight tested.

${ }^{1}$ Graduate Research Assistant, Dept of Aerospace Engineering and Engineering Mechanics, Mail Stop C0600, Member AIAA.

${ }^{2}$ Bob R. Dorsey Professor in Engineering, Dept of Aerospace Engineering and Engineering Mechanics, Mail Stop C0600, Member AIAA

${ }^{3}$ Stanley P. Finch Centennial Professor in Engineering, Dept of Aerospace Engineering and Engineering Mechanics, Mail Stop C0600, Member AIAA

${ }^{4}$ Project Manager, NASA Johnson Space Center.

${ }^{5}$ RHTF Test Director, Thermal Design Branch, NASA Johnson Space Center, Mail Stop ES3, Member AIAA.

American Institute of Aeronautics and Astronautics 
Predicting the performance of a given thermal protection system requires high-fidelity models of how the surface pyrolizes and ablates in the superhot environment behind a hypersonic bow shock. These models, in turn, require extensive knowledge of chemical kinetic rate coefficients in extreme temperature flows, information that in many cases is not available or not particularly accurate.

Various ground testing facilities can be used to partially reconstruct the reentry environment and provide empirical data against which computational models can be compared and updated. One stalwart of ablation research has been the arcjet, which heats a high pressure stream of air to temperatures on the order of several thousand Kelvin using a high-power electrical discharge before expanding the superheated flow through a supersonic nozzle. These arc-heated hypersonic facilities were used in the 1960s [1] and are still widely used today [2]-[4]. However, the use of an electrical arc discharge to heat the air has the drawback that the flow is contaminated with metal ions from the exposed electrodes.

Inductively coupled plasma (ICP) devices, which use an oscillating magnetic field within an inductor to couple energy into a partially ionized gas, do not have the problem of flow contamination. First described in 1961 by Reed [5], these devices typically involve a high-power oscillator circuit driven by a high-voltage DC supply to create an AC signal in the range of $400 \mathrm{kHz}-10 \mathrm{MHz}$ which is fed through a low-turn inductor coil surrounding a quartzencased plasma chamber. Since a metal electrode is never in contact with the gas, no metal ions are deposited into the flow, resulting in a much cleaner flow for high-enthalpy pyrolysis research. However, ICP torches have the disadvantage that they do not work as well as arc-heated wind tunnels at high flow rates or high pressures.

In recent years, several institutions have utilized ICP facilities to study how materials react to high temperature flows. The most powerful of these is the 1.2 MW plasmatron at the Von Kármán Institute [6], which has been in operation since the late 90s. Other inductive facilities exist in Germany [7], France [8], and Vermont in the US [9], and range in power from $30 \mathrm{~kW}$ to $180 \mathrm{~kW}$.

The current work involves characterizing the plasma produced by a new ICP torch facility at the University of Texas at Austin at various gas flow and input power settings. The data will be used to create an empirical model of how to set the torch to achieve desired test conditions. These measurements will be performed using standard measurement techniques like emission spectroscopy to determine a temperature profile across the radius of the torch plume, a slug calorimeter to measure the cold-wall heat flux to a test article, and a Gardon gauge to measure the radiative heat flux.

\section{Experiments}

\section{A. Plasma Torch Facility}

Experiments are performed using a $50 \mathrm{~kW}$ inductively coupled argon/air plasma torch facility at the University of Texas at Austin Wind Tunnel Labs. The torch was developed and built for UT by Applied Plasma Technologies and uses a $6 \mathrm{MHz}, 12$ $\mathrm{kV}$ AC signal to deposit up to $50 \mathrm{~kW}$ of power into up to $80 \mathrm{slpm}$ of air and/or argon. The nozzle of the torch is $30 \mathrm{~mm}$ in diameter, and the plasma chamber is $90 \mathrm{~mm}$ in diameter and $250 \mathrm{~mm}$ in length. The chamber is enclosed in a double-wall quartz tube and is water cooled. The inductor coil around the tube is also water cooled to prevent arcing between its turns.

Plasma is stabilized inside the chamber using one of two stabilization modes: direct (or forward) vortex or reverse vortex gas injection. Forward vortex stabilization is a common mode of plasma stabilization in ICP devices with net gas flow through the plasma chamber. First described by

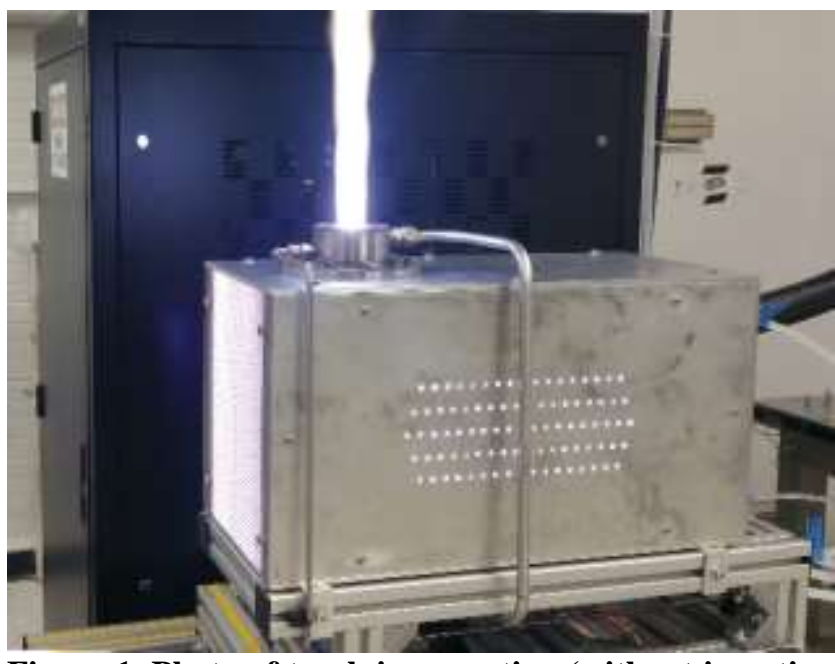

Figure 1. Photo of torch in operation (without insertion mechanism in place)

Reed [5], it works by injecting a swirling gas on the upstream end of the plasma chamber. The resulting flow rotation drives a recirculation zone in the axis of the chamber, which aids plasma propagation. Additionally, the centripetal acceleration of the rotating fluid combined with the buoyancy of the hot plasma causes the plasma to float toward the axis of the chamber, helping to insulate the chamber walls and reduce parasitic heat losses to the wall cooling system.

American Institute of Aeronautics and Astronautics 
Reverse vortex stabilization, first applied to ICPs by Gutsol [10], injects the swirling gas into the downstream end of the torch and prevents its immediate exit from the torch by means of a nozzle on the exit of the torch. In this configuration, the gas swirls around the outer radius of the chamber to the upstream end and rebounds up through the central axis of the chamber and out of the exit nozzle. This also has the effect of insulating the walls of the chamber with a cold gas, and additionally causes all gas to flow through the high temperature zone without inducing regions of recirculation. This method of plasma stabilization has been shown to be more efficient than forward vortex stabilization [10].

A unique feature of this ICP system is that the torch head is connected to the power supply using a flexible tether, which allows the torch head to be moved within a limited range. This allows the torch plume to be moved with respect to a laser diagnostic interrogation volume and therefore easily probe any arbitrary point in the flow.

\section{Traverse System}

The torch head is mounted to a three-axis traverse to allow optical diagnostic equipment to inspect any arbitrary point in the plasma flow without having to reset the often finicky alignment of laser-based diagnostic systems. The traverse uses precision ball screw actuators and servo motors to position the torch head to within $0.1 \mathrm{~mm}$ over a $150 \mathrm{~mm}$ travel range on any axis, and can be programmed to perform a timed move synchronized with a data collection system to automatically capture data from multiple points in the flow during a single run.

\section{Probe/Test Article Insertion Mechanism}

Physical probes and test articles are held in the plasma plume using a custom-built water cooled insertion mechanism. The mechanism has two arms to allow both a heat flux probe and a material specimen to be mounted at the same time and swapped into the plume quickly. In this way, the heat flux can be accurately measured immediately before inserting a test article. The arms are driven by a servo motor and can be accurately positioned to the center of the plume or scanned across the diameter of the plume to get a radial profile of flow properties. The whole insertion mechanism structure is rigidly mounted to the torch head, so that scanning the plasma plume through a laser diagnostic interrogation volume does not affect the relative positioning of the test article or physical probe within the plasma.

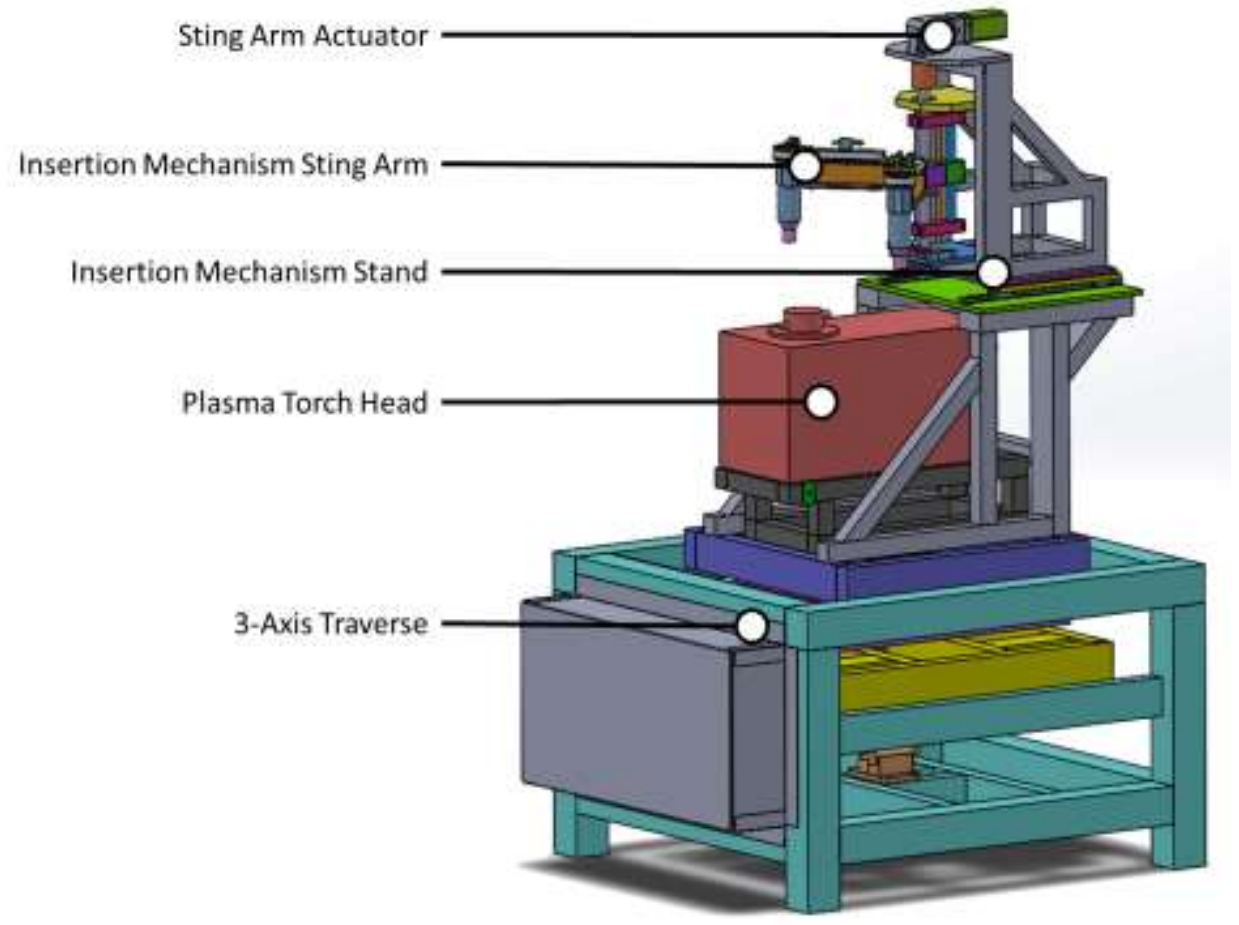

Figure 2. Model of plasma torch with insertion mechanism and 3-axis traverse.

American Institute of Aeronautics and Astronautics 
Figure 2 shows a model of how the torch, traversing table and test article insertion mechanism fit together in the assembly.

\section{B. Experimental Techniques}

\section{Heat Flux}

Cold-wall stagnation point heat flux is measured using both a Gardon gauge heat flux sensor and a slug calorimeter, pictured in Figure 3. The slug calorimeter consists of a slug of copper of known dimensions and heat capacity with a type $\mathrm{K}$ thermocouple embedded in the back surface. The slug is surrounded by, and insulated from, a copper housing such that the heat conduction within the slug can be considered 1-D. It is inserted into the plasma plume for 2 to 4 seconds and the slope of the resulting temperature curve is measured. The time rate of
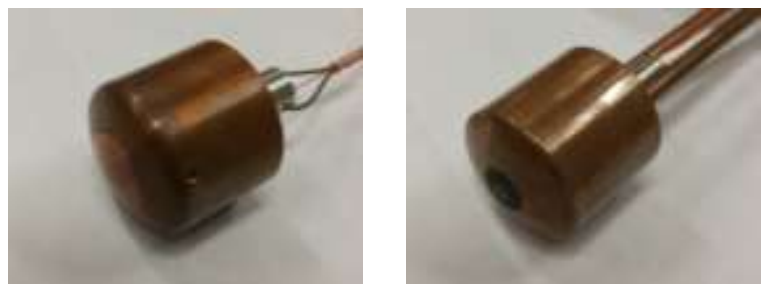

Figure 3. Left: slug calorimeter, Right: Gardon gauge

change of the temperature of the slug, $\dot{T}$, can be related to the stagnation point heat flux, $q$, by Equation 1 , where $m$, $c_{p}$, and $A$ are the mass, specific heat, and exposed surface area of the slug, respectively.

$$
q=\frac{m c_{p}}{A} \dot{T}
$$

The Gardon gauge consists of a copper body with a constantan foil disc at the stagnation point. The copper body is water cooled, providing the cold junction of the thermocouple junction between the copper and the constantan. The gauge outputs a $\mathrm{mV}$ level signal proportional to the stagnation point heat flux. Two sizes of Gardon gauge are used in the current study: a large gauge designed to be the same size and shape as the test articles to more closely match the heat flux that a test article will see and a small-diameter probe for measuring radial variation in stagnation point heat flux across the diameter of the plume.

\section{Emission Spectroscopy}

The temperature of the plasma is measured using an emission spectroscopy system. This system uses an OceanOptics HR4000 spectrometer to measure the emission spectrum between $200 \mathrm{~nm}$ and $1100 \mathrm{~nm}$, giving the sensor an effective spectral resolution of $0.44 \mathrm{~nm} / \mathrm{px}$. Light from the plasma is coupled into the spectrometer using a UV transmissive fiber optic cable. The captured spectrum is calibrated against an Ocean Optics LS-1-CAL tungsten calibration lamp to account for transmission losses in the optical system and efficiency of the CCD sensor. The spectral line broadening due to the optics of the spectrometer was estimated to be about $1.2 \mathrm{~nm}$ (FWHM) by recording a a mercury vapor lamp spectrum.

To obtain the radial variation of the plasma temperature, the collection beam was limited to a $1 \mathrm{~mm}$ wide aperture with a pencil-like field of view using an adjustable iris and a $150 \mathrm{~mm}$ focal length lens placed several focal lengths away from the torch plume, as pictured in Figure 4. Spectra

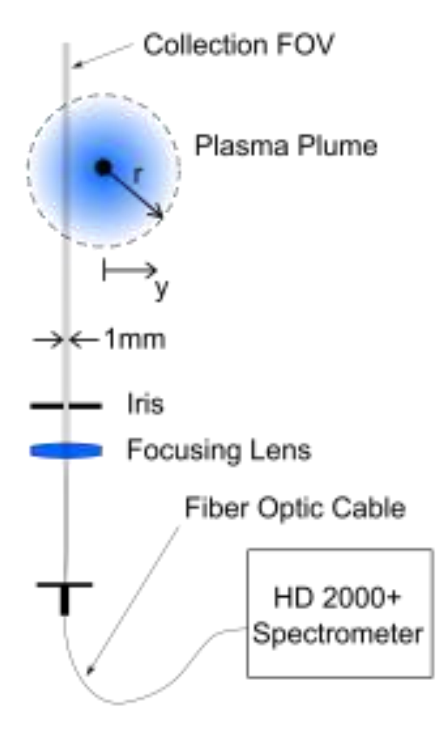

Figure 4. Diagram of emission spectroscopy collection optics were captured at y locations across the diameter of the plume at $1 \mathrm{~mm}$ intervals to obtain a spectral intensity vs position, $I(\lambda ; y)$. These intensity measurements were then Abel inverted to obtain the spectral intensity vs radial location, $I(\lambda ; r)$. The Abel inverted spectra were then used to calculate an electronic temperature as a function of plume radius.

\section{Preliminary Results}

The operating envelope of the torch using air as the test gas has been determined, and the bulk enthalpy of the gas calculated for each point within the envelope. Preliminary emission spectroscopy measurements have been taken for

American Institute of Aeronautics and Astronautics 


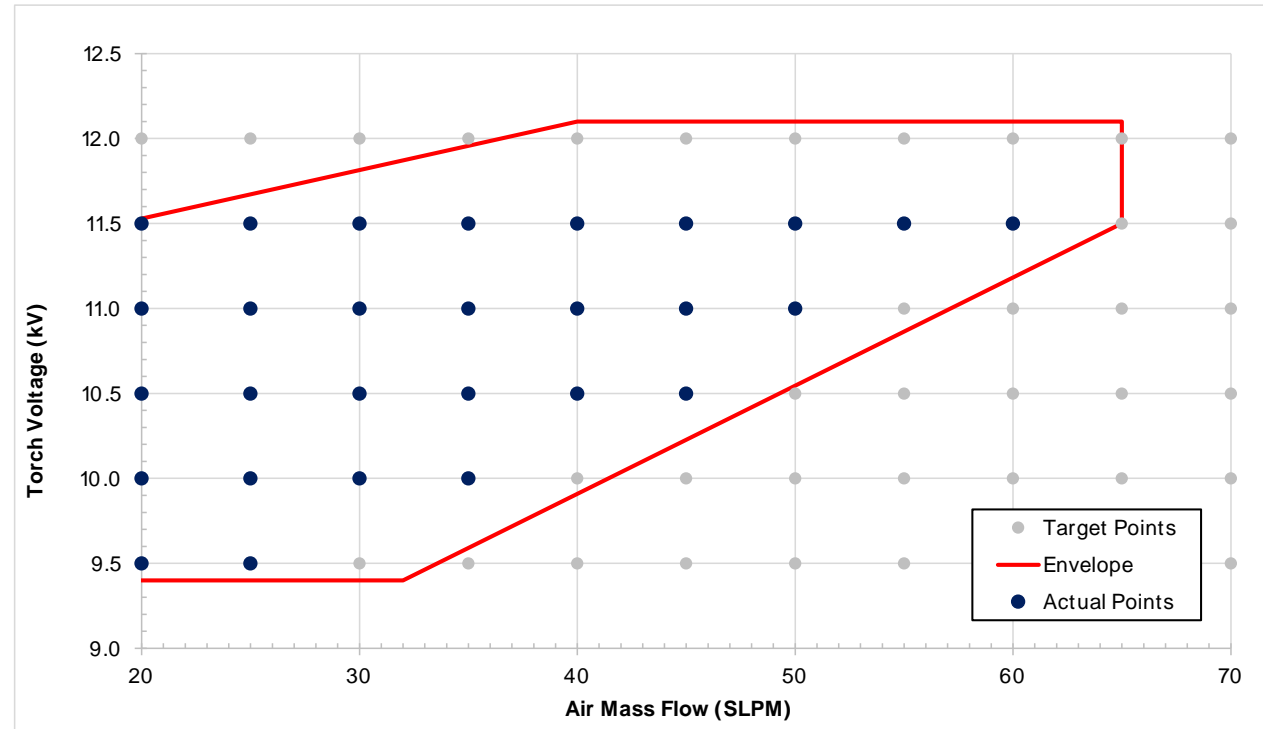

Figure 5. Operating envelope using air as a test gas.

both an air and argon plasma, and heat flux measurements have been taken over the operating envelope of the torch for two positions downstream of the torch nozzle exit.

\section{Operating Envelope}

Initial measurements of the torch included probing the operating envelope. The torch has two user inputs: the DC voltage input to the RF oscillator circuit and the test gas flow rate. So far, only air and argon have been used as test gases. The two user inputs were varied independently to determine the conditions under which the torch could achieve stable operation. The operational envelope for air is shown in Figure 5. At the low voltage end, the torch is limited by the voltage necessary to sustain gas breakdown. At the high voltage end, it is limited by the maximum allowable voltage for the circuit components. For each voltage, there is a maximum gas flow rate above which the torch cannot input enough power to sustain the ionization reactions and the plasma goes out. At the low end of flow rate, the coupling efficiency between the coil and the plasma drops and too much power gets dumped into the cooling system to allow the torch to continue to operate.

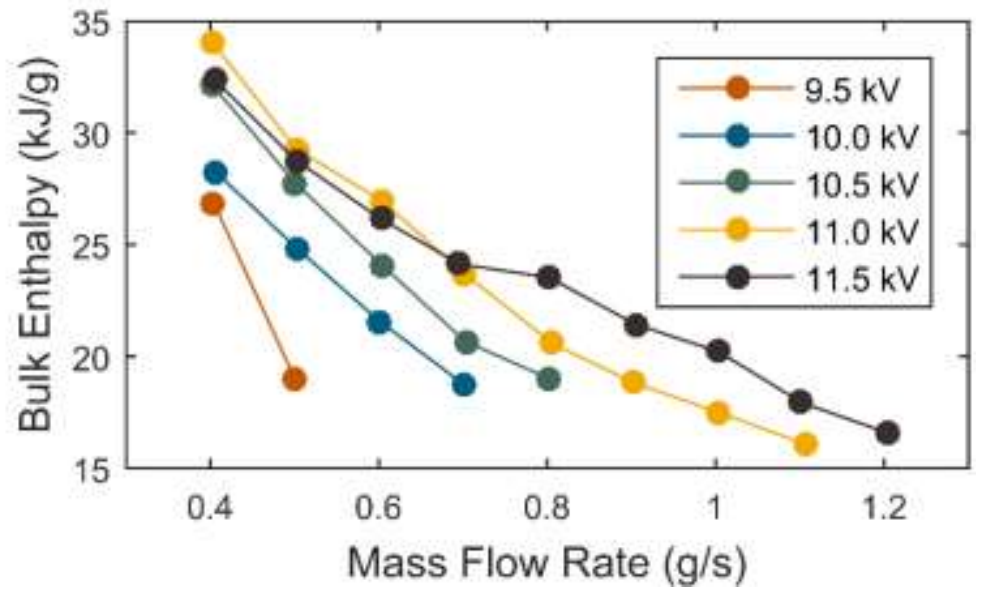

Figure 6. Bulk enthalpy of air as a function of flow rate and operating voltage

\section{Bulk Enthalpy of the Test Gas}

American Institute of Aeronautics and Astronautics 
The amount of energy deposited into the test gas by the inductor coil is estimated using the known power input into the torch and the amount of energy lost to the cooling system. It is assumed that the remainder of the energy goes into the plasma and is reported as the "bulk enthalpy" of the plasma. This value is shown for air over the operating range of the torch in Figure 6. It can be seen that the bulk enthalpy drops as the flow rate is increased. This is expected, as the voltage input is the primary driver of the plasma power. So for a constant voltage, the plasma power is approximately constant, therefore as the flow rate is increased, the bulk enthalpy decreases.

\section{E. Spectroscopic Thermometry}
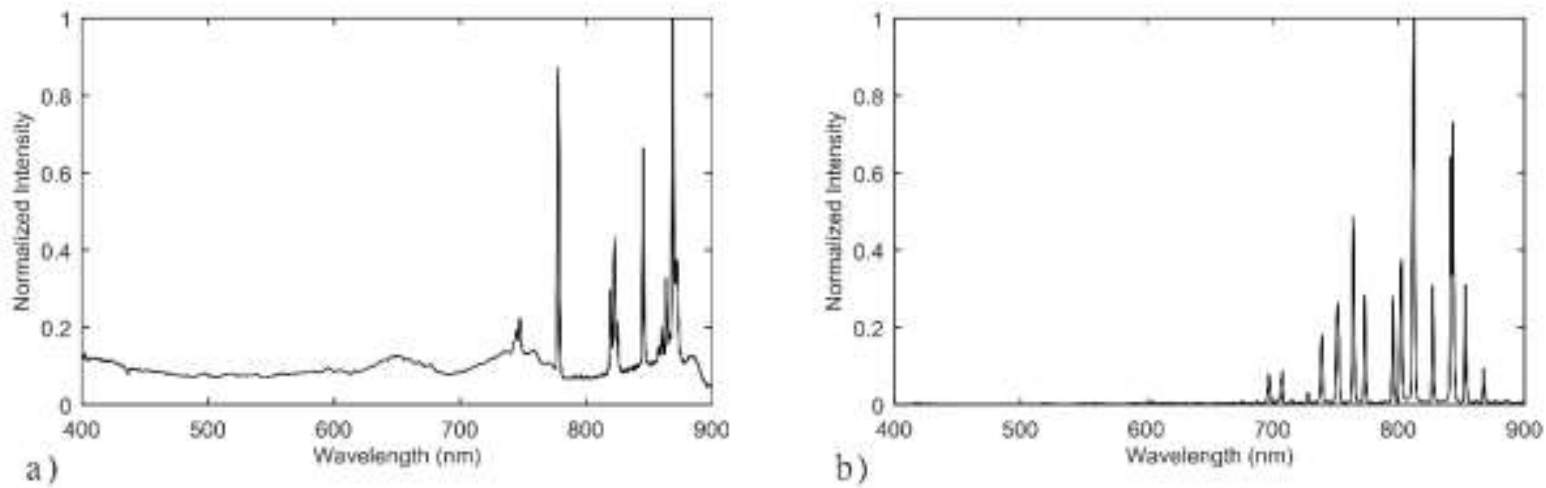

Figure 7. Sample air plasma (a) and argon plasma (b) emission spectra

Figure $7(a, b)$ shows a sample of calibrated spectra of air and argon respectively. In the air plasma emission spectrum, the dominant visible lines are the $777.3 \mathrm{~nm}$ oxygen triplet and groupings of nitrogen lines at $823 \mathrm{~nm}, 843 \mathrm{~nm}$, and $868 \mathrm{~nm}$.

The Boltzmann temperature of the air plasma is calculated from the emission spectrum using the number densities of atoms contributing to the $615 \mathrm{~nm}$ oxygen line and the $777.3 \mathrm{~nm}$ oxygen triplet. The number densities of each excited
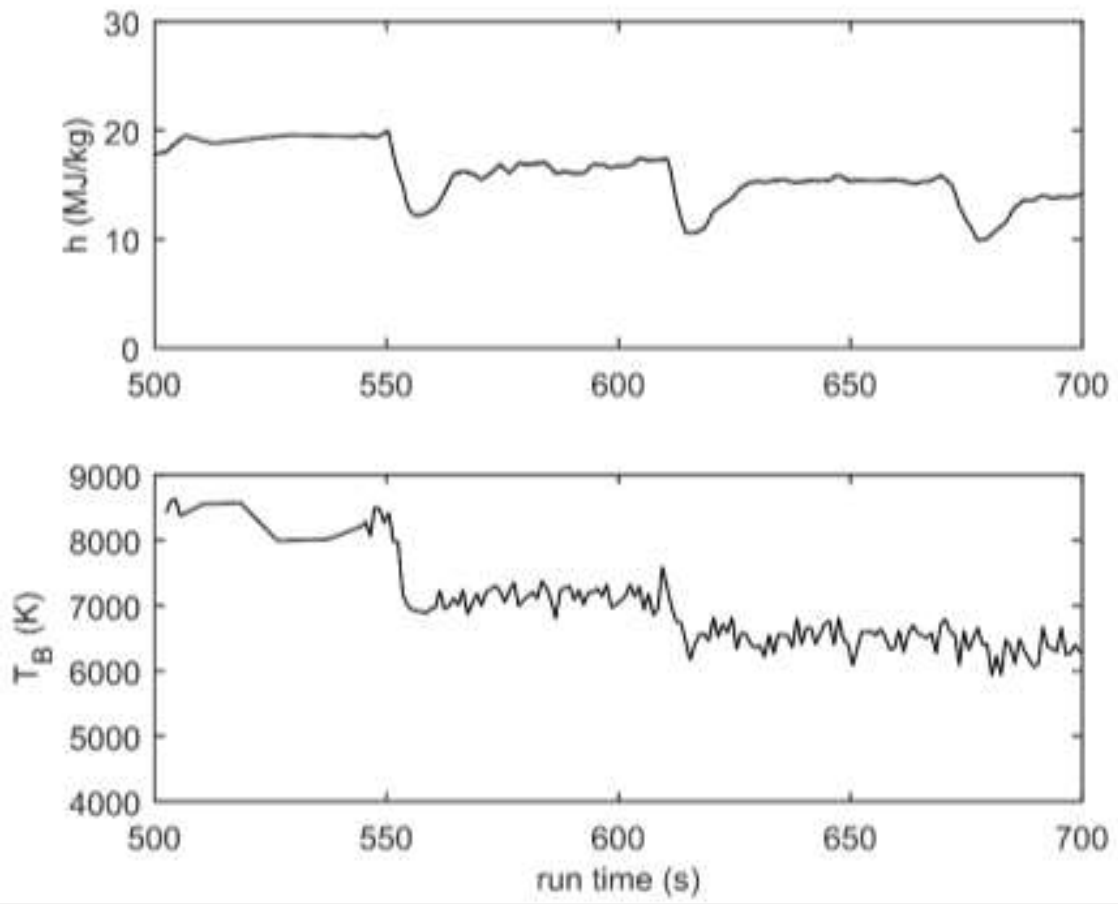

Figure 8. Boltzmann temperature plotted with specific enthalpy (h) during a run 
state are determined using Eq. (2), where the subscripts $u$ and $l$ represent the upper and lower state of the transition, respectively. The Boltzmann temperature can then be determined from the number densities in each excited state using Eq. (3).

$$
\begin{gathered}
I=n_{u} \frac{A_{u l}}{4 \pi}\left(\varepsilon_{u}-\varepsilon_{l}\right) \\
\frac{n_{u 1}}{g_{u 1}}=\frac{n_{u 2}}{g_{u 2}} \exp \left(-\frac{\varepsilon_{u 1}-\varepsilon_{u 2}}{k T_{B}}\right)
\end{gathered}
$$

Plugging Eq. (1) into Eq. (2), equates the Boltzmann temperature to the intensity ratio between the two excited states, eliminating the need for an absolute intensity measurement. Applying this equation to the spectrum in Figure 7 a yields a value of $T_{B}=6,350 \mathrm{~K}$.

The HR4000 spectrometer can be set to capture spectra at a set frequency. For one run, the spectrometer was set to record at $1 \mathrm{~Hz}$. A plot of Boltzmann temperature vs. time can be extracted from this series of spectra and plotted with the specific enthalpy to show how the temperature varies with input power. A plot of $T_{B}$ and specific enthalpy is given in Figure 8. Specific enthalpy is calculated by dividing the plasma power reported by the plasma torch control unit by the mass flow rate of gas. The torch control unit calculates plasma power by subtracting the amount of energy deposited in the torch cooling system from the system input power. Because the thermal mass of the cooling water is rather high, there is a lag time between changing the flow rate of the gas and the plasma power asymptoting to the correct value. This lag can be seen in the specific enthalpy plot as anomalous dips between each change in static level. One can see from the plot that the Boltzmann temperature in the core of the plasma plume tracks the change in the specific enthalpy very well. Taking average values for each plateau, one finds that the effective specific heat at constant pressure is approximately $2350 \mathrm{~J} / \mathrm{kg} \cdot \mathrm{K}$.

The argon plasma temperature was calculated in a more robust fashion by fitting the population of atoms in several excited states to a Boltzmann distribution. The measurement is taken in the plasma plume after the exit of the torch chamber; therefore the effect of the RF fields from the induction coil are minimal. Additionally, the flow timescales are much larger than the collision timescales, so an assumption of local thermodynamic equilibrium is reasonable.

$$
\ln \left(\frac{I \lambda}{g_{u} A_{u l}}\right)=-\frac{\varepsilon_{u}}{k_{B} T}+C
$$

The equation for the Boltzmann distribution takes the form of Eq. (4). Applying this equation to the spectroscopic data and measured intensity of the emission lines given in Table 1 and generating a linear fit produces the Boltzmann plot shown in Figure 9. From the linear fit, the temperature of the argon plasma was found to be $12,300 \mathrm{~K} \pm 1,220 \mathrm{~K}$, with the uncertainty calculated from the variation in multiple measurements.

These spectroscopic techniques were also used to calculate the temperature profile across the diameter of the torch plume by scanning a narrow field-of-view light collection system across the diameter of the plume and performing an Abel inversion. This process involves taking emission measurements from a thin, pencil-like region through the

Table 1. Spectroscopic data for Ar emission lines used in temperature calculation

\begin{tabular}{cccc}
\hline$\lambda(\mathrm{nm})$ & $A\left(10^{6} \mathrm{~s}^{-1}\right)$ & $\varepsilon(\mathrm{eV})$ & $g_{u}$ \\
\hline 675.2 & 1.9 & 14.743 & 5 \\
687.1 & 2.78 & 14.710 & 3 \\
696.5 & 6.40 & 13.320 & 3 \\
703.0 & 2.67 & 14.840 & 5 \\
714.0 & 0.625 & 13.282 & 3 \\
763.5 & 24.5 & 13.172 & 5
\end{tabular}

American Institute of Aeronautics and Astronautics 


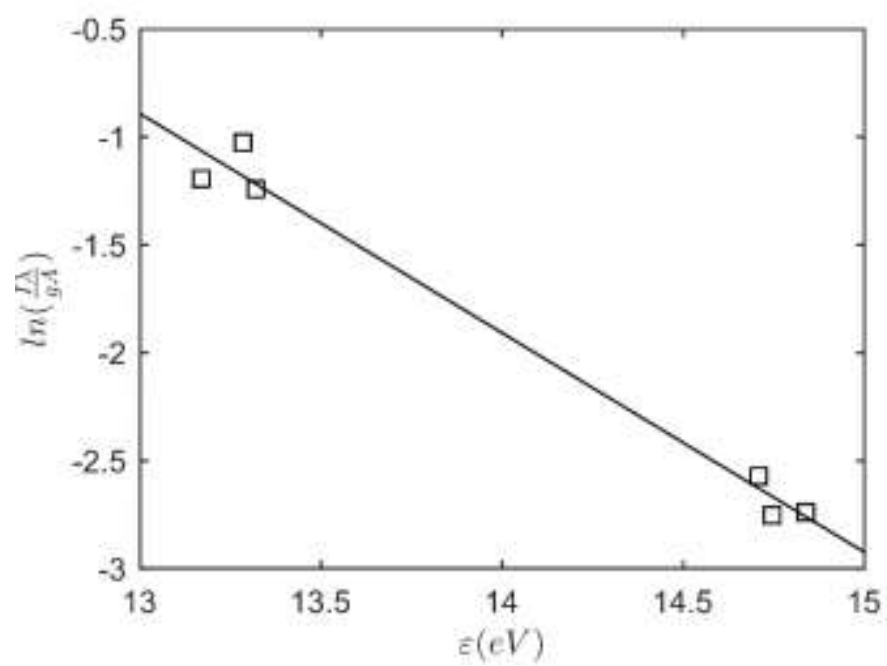

Figure 9. Sample Boltzmann plot for argon spectrum

plasma at multiple spanwise locations. Using an assumption of axisymmetry of the plasma plume properties, the radial distribution can be back-calculated from the recorded line-of-sight emission spectra.

Measurements were taken $5 \mathrm{~mm}$ downstream of the nozzle exit for two different torch settings: $10 \mathrm{kV}$ and $0.5 \mathrm{~g} / \mathrm{s}$ and $11 \mathrm{kV}$ and $0.5 \mathrm{~g} / \mathrm{s}$. The temperature profiles are shown in Figure 10. Note that the plume radius actually extends to $15 \mathrm{~mm}$, but beyond $10 \mathrm{~mm}$, the emission intensity was too weak to be measured with the current collection system. The measurements show that for a flow rate of $0.5 \mathrm{~g} / \mathrm{s}$, increasing the voltage from $10 \mathrm{kV}$ to $11 \mathrm{kV}$ increases the temperature of the gas by approximately $750 \mathrm{~K}$. For both torch settings, however, the temperature drops by $1500 \mathrm{~K}$ between the peak temperature at the centerline and the limit of the collection optics at $r=10 \mathrm{~mm}$.

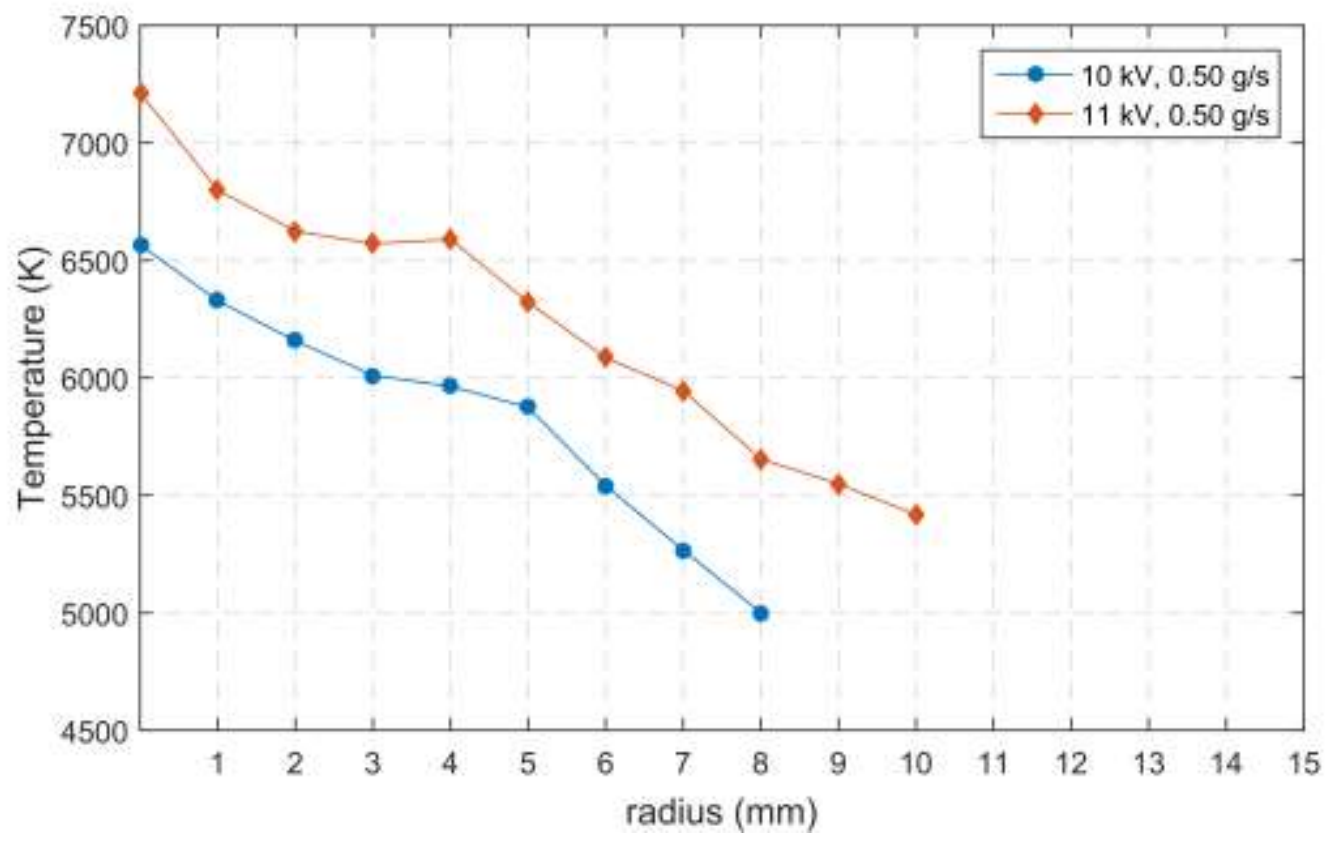

Figure 10. Temperature vs radial location in the plume for two anode voltage settings

American Institute of Aeronautics and Astronautics 


\section{F. Stagnation Point Heat Flux}

Gardon gauge measurements of the stagnation point cold wall heat flux with air as the test gas were taken at $25 \mathrm{~mm}$ and $60 \mathrm{~mm}$ downstream of the nozzle exit for the entire operating envelope of the torch. Anode voltage and test gas flow rate were varied to find a correlation between the torch settings and the measured heat flux. The results of this test campaign are shown in Figure 11 as a function of test gas flow rate.

Several interesting observations can be made from this data set. The heat flux peaks for a given anode voltage about halfway through the flow rate range, drops, and then levels off. This is likely due to the competing effects of the increasing flow velocity and decreasing bulk enthalpy as mass flow is increased for constant voltage. Also, the heat flux drops by about $30 \mathrm{~W} / \mathrm{cm}^{2}$ as the distance from the nozzle exit is increased by $35 \mathrm{~mm}$.

Similar measurements were taken at a distance of $30 \mathrm{~mm}$ from the exit of the torch nozzle using a slug calorimeter. These measurements are given in Figure 12. The change with mass flow rate for a constant voltage is qualitatively similar to that measured using the Gardon gauge. However, the measured heat fluxes are $40 \mathrm{~W} / \mathrm{cm}^{2}$ lower than those measured at a similar distance with the Gardon gauge. This is likely due to the fact that the measurement point on the Gardon gauge is approximately $3 \mathrm{~mm}$ in diameter whereas the copper slug is $15 \mathrm{~mm}$ in diameter. The latter is averaging over a larger diameter of the plume, leading to a lower measured heat flux.
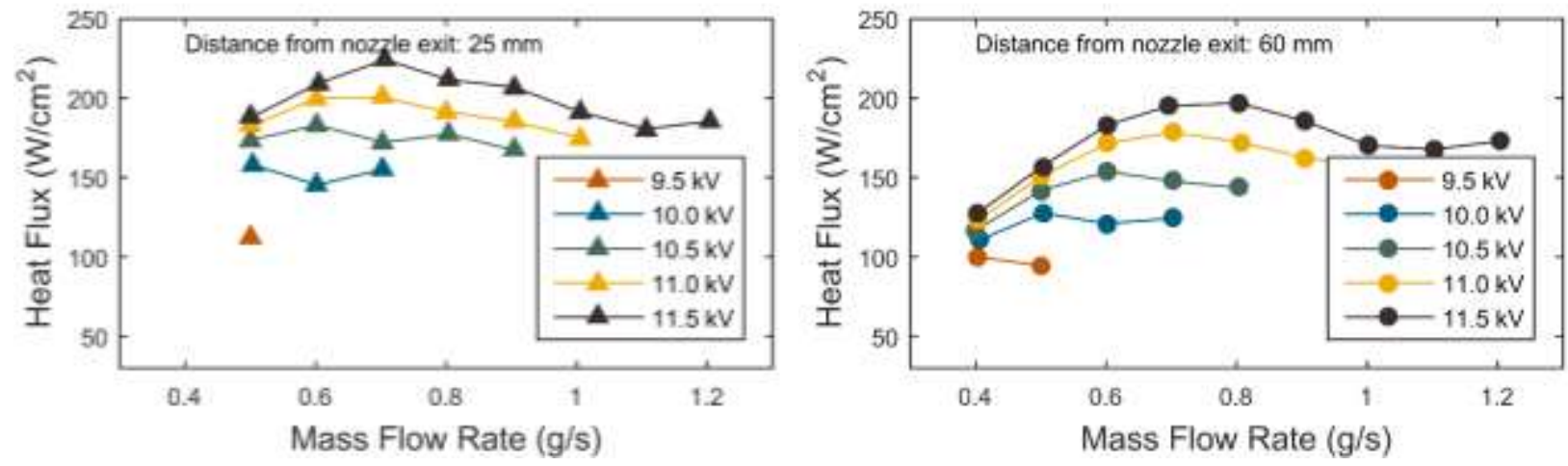

Figure 12. Stagnation point heat flux measurements from Gardon gauge.

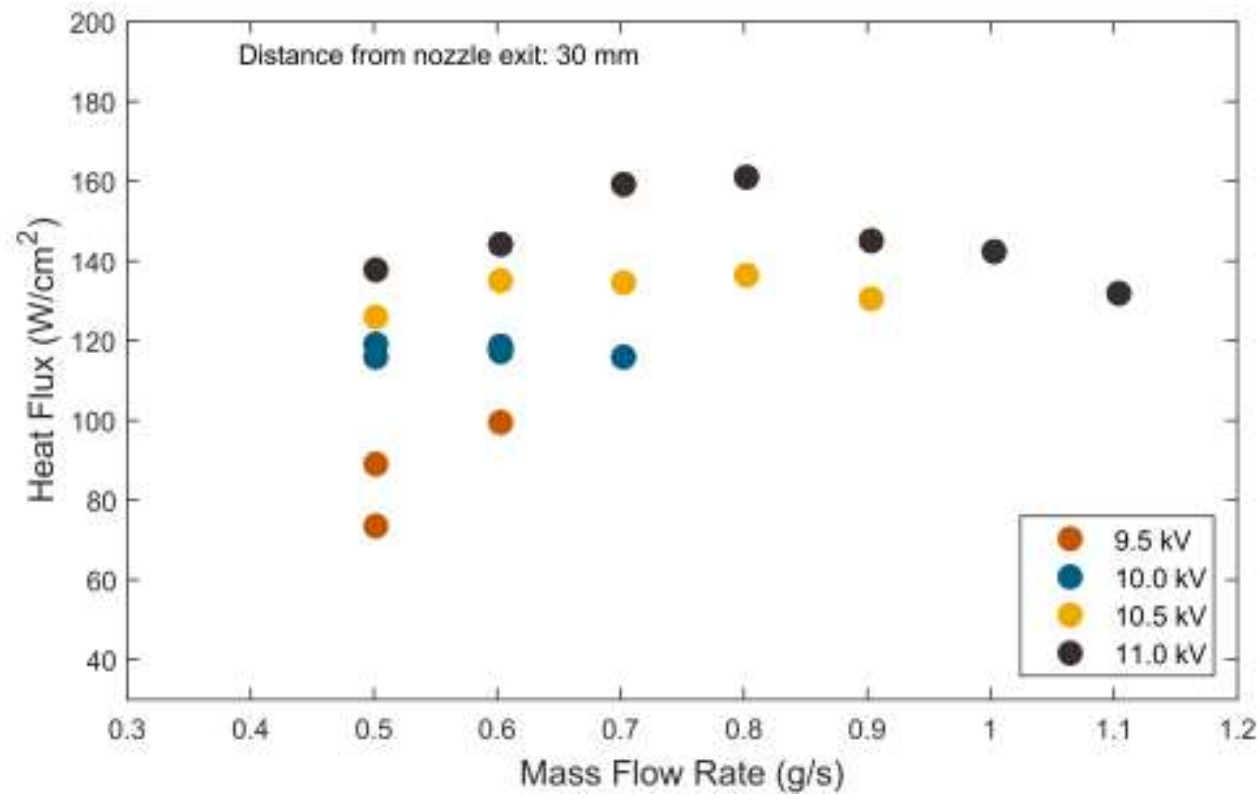

Figure 11. Stagnation point heat flux for air measured with a slug calorimeter.

American Institute of Aeronautics and Astronautics 


\section{Conclusion}

A new inductively coupled plasma torch facility at the University of Texas at Austin will be characterized using emission spectroscopy and slug calorimetry. The torch can produce air and argon plasmas, or any combination of the two, with plasma power up to $50 \mathrm{~kW}$ and a mass flow rate of up to approximately $1.6 \mathrm{~g} / \mathrm{s}$ of air or $2.3 \mathrm{~g} / \mathrm{s}$ of argon. The plasma exits the discharge chamber through a $30 \mathrm{~mm}$ diameter subsonic nozzle.

Preliminary measurements using emission spectroscopy have been performed using an OceanOptics HR4000 spectrometer. These measurements show a temperature in the air plasma of between $6,000 \mathrm{~K}$ and $8,000 \mathrm{~K}$ based on the ratio of the intensity of the $777 \mathrm{~nm} \mathrm{O}_{2}$ triplet and the $615.7 \mathrm{~nm} \mathrm{O}_{2}$ line. Measurements of the argon plasma emission spectrum give a higher temperature of about $12,000 \mathrm{~K}$.

These preliminary measurements give a good baseline for further characterization of the plasma torch. Future work will include systematic spectroscopic measurements of profiles of radial temperature and species concentration for a wide range of power and flow settings, as well as heat flux and pressure profiles using probes inserted into the plume.

\section{Acknowledgments}

This project is sponsored by NASA JSC under grant number NNX15AH17A.

Special thanks to Hai Nguyen at JSC for the design and assembly of the water-cooled insertion arm.

\section{References}

[1] R. K. Crouch and G. D. Walberg, "An Investigation of Ablation Behavior of Avcoat 5026/39M Over a Wide Range of Thermal Environments," 1969.

[2] R. Savino, M. De Stefano Fumo, L. Silvestroni, and D. Sciti, "Arc-jet testing on HfB2 and HfC-based ultra-high temperature ceramic materials," J. Eur. Ceram. Soc., vol. 28, pp. 1899-1907, 2008.

[3] T. Ogasawara, T. Ishikawa, T. Yamada, R. Yokota, I. Masayoshi, and S. Nogi, "Thermal response and ablation characteristics of carbon fiber reinforced composite with novel silicon containing polymer MSP," J. Compos. Mater., vol. 36, no. 2, pp. 143-157, 2002.

[4] B. Laub, "Use of Arc-Jet Facilities in the Design and Development of Thermal Protection Systems," in 25th AIAA Aerodynamic Measurement Technology and Ground Testing Conference, 2006, no. 3292.

[5] T. B. Reed, "Induction-Coupled plasma torch," J. Appl. Phys., vol. 32, no. 5, pp. 821-824, 1961.

[6] B. Bottin, M. Carbonaro, O. Chazot, G. Degrez, D. Vanden Abeele, P. Barbante, S. Paris, V. Van Der Haegen, T. Magin, and M. Playez, "A decade of aerothermal plasma research at the von Karman institute," Contrib. to Plasma Phys., vol. 44, no. 5-6, pp. 472-477, 2004.

[7] M. Auweter-Kurtz, F. Hammer, G. Herdrich, H. Kurtz, T. Laux, E. Schreiber, and T. Wegmann, "The Ground Test Facilities for TPS at the Institut Für Raumfahrtsysteme," in Proceedings of the Third European Symposium on Aerothermodynamics for Space Vehicles, 1998.

[8] M. E. MacDonald, C. M. Jacobs, C. O. Laux, F. Zander, and R. G. Morgan, "Measurements of Air Plasma/Ablator Interactions in an Inductively Coupled Plasma Torch,” J. Thermophys. Heat Transf., vol. 29, no. 1, pp. 12-23, 2014.

[9] W. Owens, J. Uhl, M. Dougherty, A. Lutz, J. Meyers, and D. Fletcher, "Development of a 30kW Inductively Coupled Plasma Torch for Aerospace Material Testing," in 10th AIAA/ASME Joint Thermophysics and Heat Transfer Conference, 2010, no. 4322.

[10] a. Gutsol, J. Larjo, and R. Hernberg, "Comparative Calorimetric Study of ICP Generator with Forward-Vortex and Reverse-Vortex Stabilization,” Plasma Chem. Plasma Process., vol. 22, no. 3, pp. 351-369, 2002.

American Institute of Aeronautics and Astronautics 